10 years ESJ

Special edition

\title{
Marketing Digital Como Estrategia de las Empresas de Alimentos de Villahemosa, Tabasco Frente al COVID-19
}

\author{
Miguel Ángel Ramírez Martínez \\ Universidad Juárez Autónoma De Tabasco, Mexico
}

Doi:10.19044/esj.2021.v17n4p12

Submitted: 28 October 2020

Accepted: 30 December 2020

Published: 07 February 2021

\author{
Copyright 2021 Author(s) \\ Under Creative Commons BY-NC-ND \\ 4.0 OPEN ACCESS
}

Cite As:

Ramírez Martínez M.A. (2021). Marketing Digital Como Estrategia de las Empresas de Alimentos de Villahemosa, Tabasco Frente al COVID-19. European Scientific Journal, ESJ, 17(4), 12. https://doi.org/10.19044/esj.2021.v17n4p12

\section{Resumen}

Al presentarse el COVID 19 que inicia en la segunda quincena de marzo del 2020, todos los sectores de la economía tabasqueña enfrentaron una caída de la demanda que impacta en la reducción del PIB estatal, cierre de empresas y un crecimiento del desempleo, lo que provoca un mayor deterioro de la situación económica de los empresarios y de los trabajadores, como sucedió en todo el país. Esta investigación tiene como objetivo conocer las estrategias de marketing que utilizan los empresarios del sector de alimentos para mantenerse en el mercado e identificar cómo el marketing digital ha ayudado en esta pandemia a los empresarios nuevos y a los ya existentes. Se utilizó un cuestionario con 15 preguntas; con la finalidad de tener una mayor claridad de la información y al no poder hacer las observaciones se realizaron entrevistas a profundidad a los propietarios. Entre los resultados obtenidos se encuentran: 1) Los empresarios han utilizado como estrategia el marketing digital para darse a conocer y para mantenerse en el mercado a través de las diferentes plataformas, y 2) La utilización del marketing digital a unos les ha dado buenos resultados, pero muchos están desilusionados del uso de este tipo de herramienta. Se propone que las universidades y los gobiernos locales apoyen (durante y después de la pandemia del covid 19) a los empresarios a través de la creación de plataformas "ad hoc" para la comercialización de sus productos y crear una cultura digital empresarial. 
Palabras clave: : Mercadotecnia Digital, Plataformas, Sector Alimentos, Tabasco, México

\title{
Digital Marketing as a Strategy of Villahermosa, Tabasco Food Companies Against COVID-19
}

\author{
Miguel Ángel Ramírez Martínez \\ Universidad Juárez Autónoma De Tabasco, Mexico
}

\begin{abstract}
When COVID 19 appeared, which began in the second half of March 2020, all sectors of the Tabasco economy faced a fall in demand that impacted on the reduction of state GDP, the closure of companies and an increase in unemployment, which causes a further deterioration in the economic situation of employers and workers, as happened throughout the country. This research aims to understand the marketing strategies used by entrepreneurs in the food sector to stay in the market and identify how digital marketing has helped new and existing entrepreneurs in this pandemic. A questionnaire with 15 questions was made; In order to have a greater clarity of the information and since observations could not be made, in-depth interviews were conducted with the owners. Among the results obtained are: 1) Entrepreneurs have used digital marketing as a strategy to make themselves known and to stay in the market through different platforms, and 2) The use of digital marketing has given some good results, but many are disappointed in the use of this type of tool. It is proposed that universities and local governments support (during and after the COVID-19 pandemic) entrepreneurs through the creation of "ad hoc" platforms for the commercialization of their products and create a business digital culture.
\end{abstract}

Keyword: Digital Marketing, Platforms, Food Sector, Tabasco, Mexico

\section{Introducción}

El COVID 19 que inicia en China en a finales del 2019 llega a México en marzo del 2020. Tabasco, un estado localizado en sureste de la república mexicana, con una población de 2'975,272 habitantes de acuerdo a la encuesta intercensal 2015 del INEGI, inicia la primera cuarentena en marzo del 2020, con la suspensión de actividades en la mayor parte de los sectores económicos del estado.

La crisis psicológica que se vive en cada uno de los diferentes contextos de la sociedad se va incrementando a medida que crece el número de infectados y el número de muertes, aunado a la psicosis ocasionada por la falta de medicamentos y de camas en los diferentes hospitales de los 17 
municipios que integran el estado de Tabasco. En este contexto casi el 100\% de las actividades económicas se paralizaron.

López, R. (20 de marzo de 2020) menciona: En un mensaje en cadena estatal el gobernador, Adán Augusto López Hernández instruyó el cierre de restaurantes, bares, cantinas, cafés, de las playas en el estado, así como la prohibición de reuniones masivas como medidas de prevención ante los casos de Covid19 detectados en la entidad. Asimismo, indicó que su gobierno ha dictado las medidas necesarias a fin de garantizar en Tabasco el abasto racional de víveres y suministros, por lo que dijo "son innecesarias" las compras de pánico".

En mayo, la situación del estado ${ }^{1}$ y del municipio se vuelve más caótica, por lo que las autoridades deciden reducir las restricciones. Pérez, J. (6 de mayo de 2020) de El Heraldo de Tabasco señala: Únicamente bajo el esquema de ventas a domicilio es como pueden operar el fin de semana los 2 mil restaurantes instalados en la ciudad de Villahermosa, que no pueden recuperarse de la caída de ingresos que les signifique la inactividad el Día de las Madres. De acuerdo a la presidenta de Cámara Nacional de la Industria de Restaurantes y Alimentos Condimentados (CANIRAC) Violeta Isidro Pérez, esto significa una caída aún más drástica de lo que llevaban durante la contingencia por el Covid 19, aunque aclaró que no se tienen contabilizadas las pérdidas económicas. De los más de 2 mil restaurantes afiliados a CANIRAC Tabasco, algunos se han mantenido trabajando bajo el esquema de ventas con servicio a domicilio, y otros más han optado por cerrar, aunque de cualquier forma están afectados, ya que el fin de semana no pueden abrir y que los que hacen solos pueden vender a domicilio.

A medida que transcurrían los meses, la situación económica de la localidad empeoraba. Alejo, H (12 de junio de 2020) del noticiero de la XEVA, señaló: La presidenta de la Cámara Nacional de la Industria Restaurantera y Alimentos Condimentados (CANIRAC) en Tabasco, Violeta Isidro Pérez estimó que al menos un 15 por ciento de los restaurantes, bares, fondas y otro tipo de establecimientos del estado, no volverán a abrir una vez que concluya la contingencia por el COVID19, por la crisis económica que la pandemia les dejó. Indicó que actualmente los bares han estado cerrados desde que comenzó la contingencia por el Coronavirus y opinó que muchos posiblemente no podrán volver a abrir, si no reciben algún tipo de crédito o apoyo económico. Sin embargo, aclaró que será hasta después del día 22 de este mes, si el semáforo de contagios del estado cambia, que se sabrá con

${ }^{1}$ A finales del siglo XX e inicios del siglo XXI la economía tabasqueña depende más del sector secundario, al pasar la aportación al PIB de este sector del $29.8 \%$ en el 2000 a $63.8 \%$ en el 2010; una caída del sector primario del 6.8\% del PIB en el 2000 al $1.6 \%$ en el 2010 y una caída del sector terciario del 64\% en el 2000 al 34.6\% del PIB en el 2010. 
precisión cuántos establecimientos sí pudieron volver a abrir. Por último, señaló que se tendrá que hacer un estudio de mercado para saber con exactitud cuántos establecimientos abrieron, cuántos no y el número de empleos que se perdieron.

La situación que vive el estado en sus primeros seis meses de pandemia no solo ha impactado a los empresarios y al personal que trabajaba en dichos lugares, sino también ha traído un efecto multiplicador en todos los sectores que forman parte de las cadenas de valor de cada uno de los sectores de la economía.

Ante este esquema es importante conocer la situación en la cual se encuentran los empresarios de los distintos sectores de la economía. Debido a la importancia que tiene el sector de preparación de alimentos, tanto por el número de establecimientos como por el número de empleos que general, se ha decidido llevar a cabo esta investigación en el sector restaurantero, específicamente la forma de como comercializan su producto.

El Marketing digital Identifica las necesidades para convertirlas en demandas utilizando todos los mecanismos bidireccionales a su alcance para establecer una relación próxima entre quien oferta y quien consume. Sus estrategias de comunicación pretenden que la transmisión de la información se realice de manera multidireccional. Es decir, la organización permite que el potencial cliente interactúe con su mensaje, lo que añade valor a la situación comunicativa y al producto. Es realmente un conjunto de tácticas que abarca desde la creación de banners, email marketing, folletos digitales hasta el desarrollo de sitios web. Su objetivo es conocer las necesidades del cliente mediante el dialogo y aportarles una solución. (págs. 14-15).

De acuerdo con Sixto (2016, p.17) dentro de las redes sociales y las nuevas tecnologías, el marketing digital tiene principalmente tres objetivos:

1) Conseguir una comunidad de clientes o seguidores cada vez mayor para la empresa o marca

2) Que esa comunidad sea conformada por clientes reales y habituales, con confianza en la empresa, y

3) Fomentar o crear la vinculación de los clientes con esa marca.

Sixto (2016, págs. 18-19) señala que el marketing digital se basa en lo que se conoce como las 4Fs: flujo, funcionalidad, feedback y fidelización.

- Flujo: el usuario o cliente debe sentirse atraído por la página para poder captar su atención y que no abandone el sitio en el que se intenta que permanezca: Para ello se requiere un contenido llamativo y de calidad.

- Funcionalidad: navegar por el sitio web debe ser fácil y sencillo, sin que haya riesgos de que el usuario se acabe perdiendo y abandone la página.

- Feedback o retroalimentación: el internauta o usuario debe notar que existe una respuesta a sus dudas o preguntas, al igual que si estuviera 
hablando con un dependiente en la tienda. Con esto también mejoran la reputación y la confiabilidad de la marca.

- Fidelización: Es necesario que una vez que el usuario se haya interesado por la marca encuentre motivos para seguir interesándose más y crear un compromiso entre ambos.

Así mismo Sixto (206: p.19) menciona que entre las principales ventajas que presenta el uso del marketing digital están:

$\checkmark$ Resultados medibles casi a tiempo real.

$\checkmark$ Poder viral.

$\checkmark$ Bajos costos de producción

$\checkmark$ Presupuestos flexibles.

Con lo anterior podemos señalar que ambos marketing son complementarios, el tradicional y el digital.

Las plataformas digitales se pueden concebir como un sistemas o sitios web personalizados, que satisfacen una o varias necesidades específicas en beneficio tanto del usuario, como de la empresa. Estas usan la infraestructura del internet para comunicarse entre ellas y de esta forma construyen una red con un propósito específico, así que cuando nos referimos a sitios web2.0 nos referimos a plataformas digitales con propósitos específicos para agilizar los servicios que brindan.

De acuerdo a Meneses (2017) en el documento intitulado: La importancia de una plataforma digital alineada a tu negocio, clasifica a las plataformas de la siguiente forma:

1) Plataformas de administración de contenido

Hoy en día la clave de un buen sitio web radica en la capacidad de brindar contenido relevante y actual para nuestras audiencias, si pensamos en un sitio web como una tienda de moda, sabemos lo importante que es mantener nuestras vitrinas con lo más reciente de las colecciones o bien con grandes rótulos de "Rebajas" en épocas de liquidación. Sin este tipo de plataformas, la única forma de lograr tener el sitio actualizado periódicamente seria con un equipo de programadores en nuestra planilla a tiempo completo, pero gracias a que hoy en día contamos los "CMS" o Content Management Systems tenemos la capacidad de crear estrategias de contenido con total autonomía en nuestro propio entorno digital.

2) Plataformas para envío de correos

Los newsletters o boletines electrónicos son un pilar del marketing online que nos permiten llegar a nuestros clientes. Nuestro correo electrónico personal o de negocios no nos permite esta titánica tarea. Usar estas plataformas tiene una gran variedad de beneficios, los usuarios no ven los correos de otras personas a las que se les envía el comunicado, la interacción con cada parte del boletín puede ser 
medida, es un formato liviano y digital por lo que su costo de envío es significativamente bajo, además podemos segmentar audiencias según parámetros demográficos y más.

3) Plataformas de e-commerce o comercio electrónico

La buena noticia es que si deseamos vender nuestros productos en línea no tenemos que empezar de cero. Existen muchas plataformas para vender productos, ya sea a través de sitios especializados donde podemos crear nuestra propia tienda, o bien servicios que nos permiten incorporar esta estructura de negocios a nuestro sitio web. Muchas, sino la mayoría, ya tienen incorporados sistemas para pagar en línea como Paypal, lo que generará mayor confianza en los compradores ya que saben que su información permanecerá segura.

4) Plataformas de colaboración

Estas herramientas son muy populares hoy en día, sirven para compartir en la nube grandes cantidades de información y crear accesos rápidos para compartir con quienes deseemos. Un buen ejemplo es Dropbox, donde tenemos entre 2 a 5 gigas de espacio gratis en un sistema que nos permite controlar las versiones de nuestro trabajo, así como guardar stocks de fotografías y otros recursos que normalmente necesitan mucho espacio de almacenamiento. Otra plataforma que nos ofrece la facilidad de colaboración son las diferentes herramientas de Google donde varias personas pueden trabajar al mismo tiempo en un documento que está en la nube.

5) Plataformas de socialización o Redes Sociales

Estas plataformas han venido a redefinir por completo las prácticas de marketing donde un buen diálogo nos permite usar a nuestra audiencia como principal medio para difundir nuestros mensajes. Cada empresa tiene sus propias necesidades de comunicación y la plataforma que le sirve a unos no genera los mismos resultados para otros, esto se debe a que cada red social tiene una dinámica y un propósito específico en nuestra mezcla de marketing:

5.1 Facebook es un sitio diseñado para crear conversaciones y compartir contenido de forma sencilla.

5.2 Twitter es considerado un microblogging (140 caracteres), está orientado a mensajes vivenciales y sirve muy bien para eventos o noticias que suceden al momento.

5.3 Instagram (Una imagen dice más que mil palabras) la aplicación está totalmente orientada a teléfonos y a una experiencia táctil. 


\section{Metodología}

Es una investigación de tipo exploratoria Ante la pandemia del COVID 19 que se vive en el estado de Tabasco país de no poder estar físicamente en los negocios para verificar los datos como lo exigen los métodos científicos se utilizó la encuesta telefónica y se entrevistas a profundidad. Se eligieron para esta investigación a los negocios que prestaban servicio de alimentos durante estos últimos meses (marzo-agosto 2020), los cuales fueron identificados a través de redes sociales, periódicos y radio; con los datos obtenidos, nos comunicamos vía telefónica para solicitarle responder a un cuestionario de 15 preguntas, que se les envió por teléfono móvil, que nos permitirían entender el comportamiento mercadológico de los negocios ante esta pandemia. Estas preguntan eran: 1) Identificar si los negocios prestaban sus servicios antes de la pandemia; 2) Los motivos que les hicieron emprender el negocio; 3) El conocimiento que tenían sobre el negocio; 4) La inversión que realizaron para iniciar el negocio; 5) Las formas como dan a conocer el negocio; 6) En que plataformas se anunciaban; 7) El costo o la inversión que habían realizado para anunciarse; 8) La forma como enviaban el producto al comprador; 9) El número de empleados directos que tenían; 10) Si estaban dados o no de alta en el SAT; 11) Pagaban una renta o se localizaban en un lugar de su propiedad; 12) Contrataron una persona para que les apoyar en la publicidad y colocarlo en una plataforma o lo hicieron ellos mismos; 13) Sabían cómo funcionaba la plataforma donde se anunciaban; 14) Consideraban que la inversión que hacían en la plataforma era rentable y 15) Al terminar la pandemia seguirían utilizando la plataforma actual o buscarían otras.

Se utilizó la estadística descriptiva para hacer la clasificación de los datos obtenidos y se realizó la entrevista a profundidad para poder aclarar algunos aspectos que los datos estadísticos no la proporcionaban. Debido a la situación que se vive resultó muy difícil tener entrevista a profundidad con todos los propietarios de los negocios, por lo que solo se realizaron 11.

\section{Resultados}

Se tomaron de las distintas plataformas información de los restaurantes, así como de la publicidad presentada en radio y periódicos del estado de Tabasco, explicando a los propietarios y/o administradores la importancia que existía de conocer cómo la población restaurantera estaba enfrentando la pandemia del COVID 19, se les suministro un cuestionario. De un total de 90 cuestionarios enviados solo 47 dieron respuesta (22 ya existían antes de la pandemia y 25 se crearon a partir de abril del 2020), Los datos recabados se clasificaron en dos grupos: a) los que ya existían antes de la pandemia y b) los que se fueron creando durante la pandemia. 
La información obtenida de los cuestionarios aplicados a las empresas mostró los siguientes resultados.

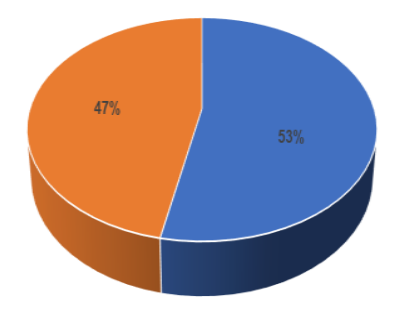

Del total de negocios que resolvieron el cuestionario 22 de ellos se instalaron en los primeros dos meses de la pandemia y 25 ya existían, pero su principal actividad era prestar atención al público en sus instalaciones (Figura $1)$.

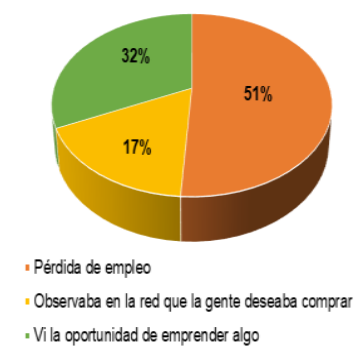

La figura 2 nos muestra que la mayor parte de negocios son resultado de la pérdida de empleo.

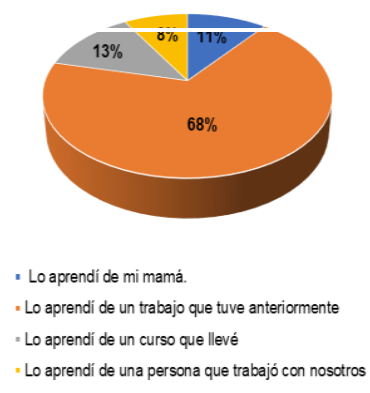

La figura 3 muestra que el conocimiento previo del negocio que emprenden porque lo aprendió en un empleo anterior. 


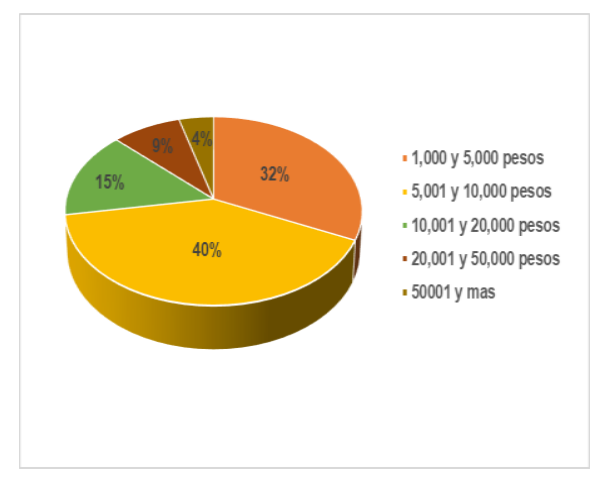

La mayor parte de los que emprendieron un negocio (figura 4) lo realizaron con una inversión entre $\$ 5,000$ y $\$ 10,000$ pesos.

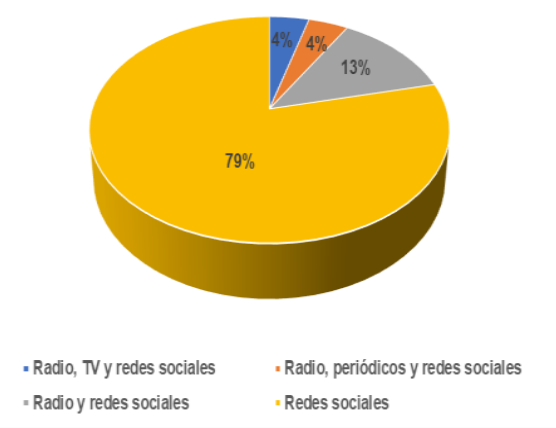

La figura 5 nos muestra que la mayor parte de los negocios se dan a conocer a través de redes sociales.

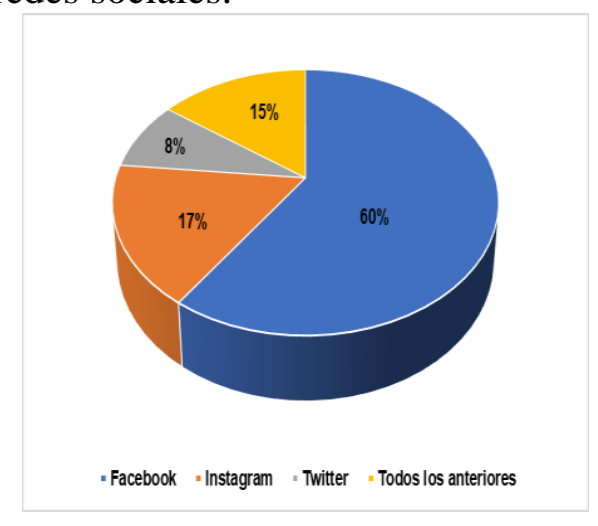

La plataforma más utilizada por los empresarios en esta investigación es Facebook (Figura 6). 


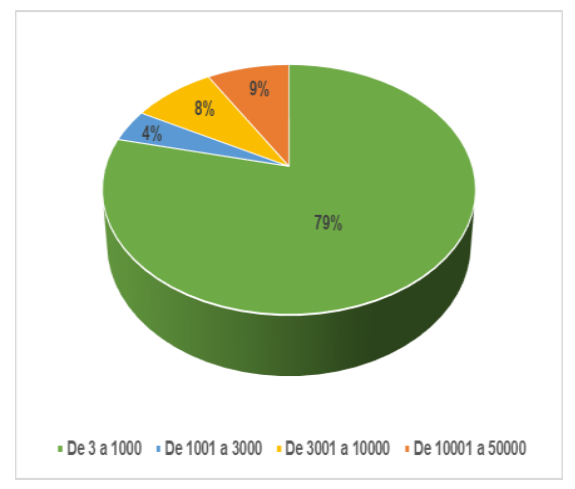

El gasto en publicidad y propaganda, de la mayor parte de los encuestados, oscila entre los $\$ 3$ y $\$ 1,000$ pesos (Figura 7).

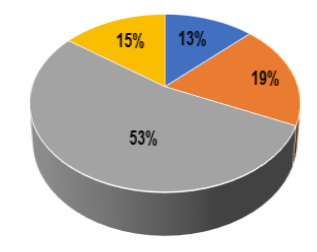

$$
\text { - Entrega directa en el negocio }
$$

- Repartidores contratados sin transporte

- Repartidores contratados con motocicleta

- Uber o taxis

La mayor parte de los propietarios de restaurantes utilizan repartidores contratados con motocicletas para entregar sus productos (Figura 8).

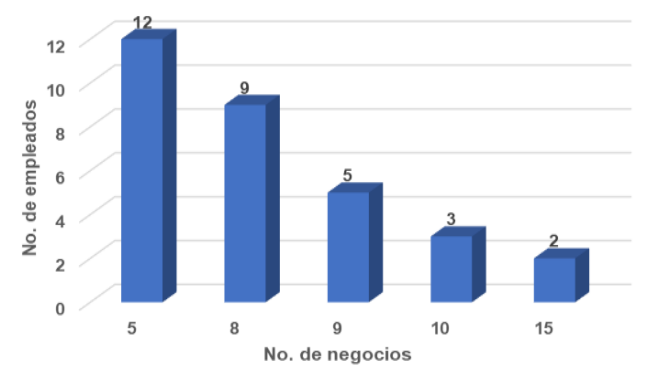

Dos empleados tienen un tercio de los establecimientos encuestados y entre dos y tres empleados más del 50\% de los establecimientos (Figura 9). 


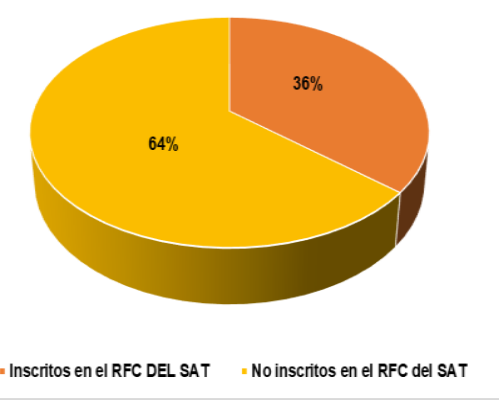

Un tercio de los negocios encuestados son informales (Figura 10).

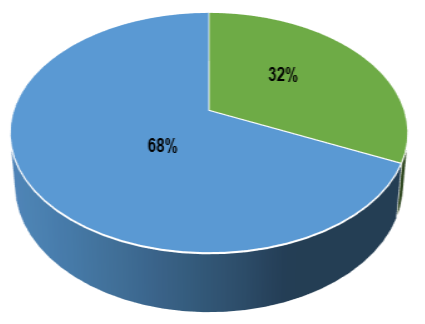

- Pagan una renta - Local propio

Dos terceras partes de los negocios encuestados se localizan en un área de su propiedad (Figura 11).

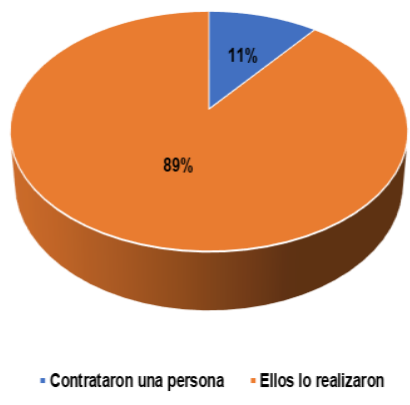

En la mayoría de los establecimientos los propietarios o uno de sus familiares realizaron la publicidad (Figura 12). 


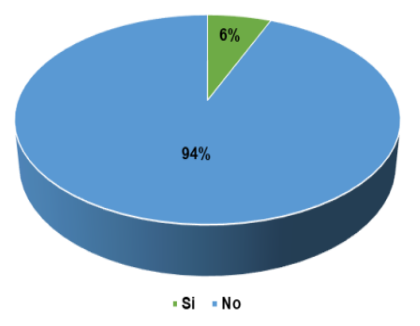

La mayor de los propietarios y/o encargados de los establecimientos no conocen cómo funcionan las plataformas (Figura 13).

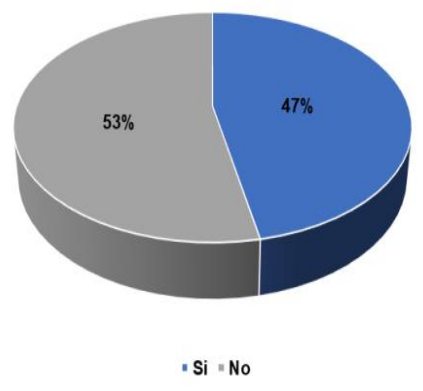

La mayor parte de los propietarios que han invertido en alguna plataforma consideran que no es rentable (Figura 14).

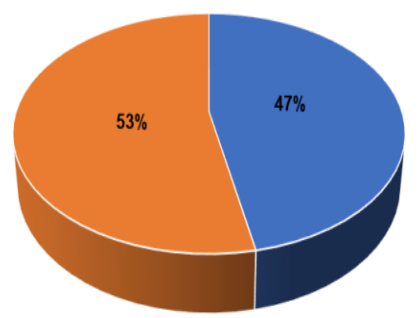

- Si $=$ No

La mayor parte de los propietarios que han invertido en una plataforma no lo volverían hacer después de la pandemia (Figura 15).

\section{Discusión}

Entender el comportamiento empresarial en una época de pandemia en cualquier parte del mundo es complejo por el estado de ánimo en el cual se encuentra el empresario, esto depende de varios factores (edad, sexo, situación familiar, personalidad, etc) y no solo de la situación económica en que se 
encuentre la empresa en el momento. La planeación, en estos casos, es casi imposible por el alto grado de incertidumbre que existe, debido a que carece de elementos que le permita una visión clara sobre su futuro.

Los datos obtenidos en esta investigación nos muestran que ante situaciones generadas por la pandemia de COVID 19, los empresarios tienen diferentes comportamientos, algunos emprenden un nuevo negocio con el fin de obtener los recursos necesarios para su subsistencia y generalmente lo realizan en un área de la industria que conocen o que han estado en ella durante un tiempo determinado. Lo que podría corroborar lo que señala Pico (2016, p. 14): El emprendimiento por necesidad consiste en materializar una idea de negocio en la cual no necesariamente hay potencial crecimiento. Nace de la motivación por crear una solución a una problemática que acontece en un periodo de tiempo determinado y bajo unas circunstancias específicas. Es así que se ejecuta una idea, sin el total conocimiento de su potencial en el mercado, dando paso en algunas ocasiones a una aventura empresarial. Sin embargo, la realidad actual conlleva en su análisis que un emprendimiento bien ejecutado podría convertirse en negocio potencial con muchas oportunidades que lo lleven a posicionarse en el mercado y por ende alcanzar éxito empresarial

Siguiendo a Pico, podríamos pensar que los negocios, del sector de alimentos, que han sido creados durante la pandemia del COVID 19, en Villahermosa, Tabasco., son resultado de la motivación que han tenido los emprendedores para darle solución a la problemática de satisfacer la demanda de alimentos que existe en este periodo, al darse cuenta de que muchos medianos y/o grandes negocios han cerrado ante las normas que les impide seguir funcionado.

Se podría señalar que la pandemia provocó un incremento del emprendedurismo, se generan negocios a partir de las demandas que identifican los emprendedores en las redes sociales. Frente una serie de restricciones que ponen los gobiernos locales ante el riesgo de tener un incremento en el número de infectados, dichos negocios se realizan de manera virtual a través de las redes sociales, que hoy más que nunca juegan un papel determinante para dar a conocer un producto y se conozca entre las distintas comunidades de cibernautas que existen en dichas redes, así como a los diferentes tipos de usuarios de redes sociales, que de acuerdo al banco Firs Direct, los clasifica en :Ultras, Dippers, Deniers, Vírgenes, Lurkers, Peacocks, Ranters, Changelings, Ghosts, Quizzers, Informadores, y los Approval seekers, quienes finalmente se pueden considerar en la lógica del mercado virtual como oferentes y/o demandantes, así pasamos de un marketing tradicional a un marketing digital.

Tanto los emprendedores que se dan frente a la pandemia como los negocios que ya existían hacen uso de las redes y de las distintas plataformas 
que existen. Los datos muestran que la mayoría de los propietarios de negocios desconocen el funcionamiento técnico del funcionamiento de cada una de las plataformas, pero ante la necesidad de darse a conocer y vender su producto intentan introducirse en ellas. La mayoría colocan su publicidad (tweter) sin entender a quienes les llegara, ya que lo hacen de una manera "practica", escuchando los consejos de alguien o simplemente "jugando" con su teléfono celular y la plataforma para insertarla, pero los algoritmos de las redes sociales reducen el alcance orgánico de dichas publicaciones. Otros intentan contratar el servicio de la plataforma "apostando" cierta cantidad monetaria que le cobra la plataforma por cada una de las personas que ven y aceptan la información, es decir, la plataforma segmenta los mercados y les señala claramente quienes podrían ser los perfiles que mejor se ajustan al producto que están promocionando. De esta manera llegan a clientes potenciales y pueden interactuar con ellos. El costo de dicha campaña va a depender de varios factores, pero principalmente del número de clientes potenciales a los cuales lleguen.

Los datos del trabajo arrojan que 25 de los 47 encuestados no están dispuestos a seguir invirtiendo en las redes sociales, al cuestionarles por qué, su respuesta es que no obtuvieron los resultados que esperaban, en otras palabras, están desilusionados de su inversión. Al hacer un cuestionamiento más profundo sobre dicho aspecto señalan que no conocen como funciona la plataforma y que esperaban que al igual que muchos les iría bien; pero al preguntarles si sabían que porcentaje de los que invertían en ellas les iba bien, contestaban que no, por lo que ha sido la "necesidad" o la "ignorancia" lo que los ha llevado a invertir en este tipo de marketing.

Existe un gran desconocimiento del uso de las plataformas por parte de los dueños de las empresas. Solo cinco de los 47 contrataron una persona especializada que les ayudó en el proceso de integración de su empresa a la plataforma. Los cinco opinaron que si es rentable su utilización. Una de las preguntas más frecuentes que existen por parte de los restauranteros, en los cuestionamientos a profundidad, es si deberían usar todas las plataformas a la vez y nuestra respuesta fue que depende de las necesidades que su empresa tenga; que deben considerarse el costo que implica la utilización de cada una de ellas y la cantidad de dinero que desean invertir los empresarios.

A pesar de que los 47 empresarios han utilizado un formato de publicidad de sus empresas donde ofrecen sus diferentes productos que desean comercializar a través del uso de esquemas, fotografías, precios, tiempos y tipos de entrega, entre otros y los han integrado a las diferentes redes sociales (marketing digital), solo algunos han logrado sus objetivos. La mercadotecnia tradicional pensaría que el problema podría haber sido la información, los medios o el producto; hoy, ante esta nueva realidad cibernética sabemos que el problema central es la forma como se "sube" esta publicidad a la plataforma. 


\section{Conclusiones}

Esta investigación nos permite conocer que ante el COVID 19 las MIPYMES restauranteras de la ciudad de Villahermosa, Tabasco., enfrentan esta pandemia con estrategias del marketing digital, el cual ha cobrado una mayor importancia ante la situación que vive el estado y el país, así como por la cantidad de teléfonos móviles que permiten una comunicación permanente a través del uso de las diferentes plataformas que existen en el mercado.

La estrategia de marketing digital que los empresarios ocupan es una combinación del marketing tradicional con el marketing digital. En cuanto al primero, utilizan estrategias de precio, promoción, plaza, producto y servicio combinando estas con las estrategias de marketing digital: Diseño de página digital, plataforma y retroalimentación.

En esta pandemia surge un empresario emergente que desconoce tanto las estrategias del marketing tradicional como del marketing digital, en donde se requieren decisiones rápidas que den respuestas a las demandas emergentes, por lo que algunos se ven en la necesidad de contratar a un individuo especializado para que elabore su marketing y acceder a una plataforma o en su defecto elaborarla por ellos mismos y difundirla a través de sus redes sociales. En el primer caso dependerá de la inversión que desee hacer con lo que le garantizaría una mayor probabilidad de llegar a una mayor audiencia; en el segundo, la inversión será menor pero la probabilidad de fracaso será mayor.

El utilizar las redes sociales no significa que los empresarios las saben usar, tampoco que garanticen que habrá un incremento de sus ventas por la difusión entre los miembros de esa comunidad de cibernautas, tampoco tiene un conocimiento cierto de que quienes, vean, lean o conozcan su producto o servicio lo adquirirán. Para la mayor parte de la población el funcionamiento técnico de las plataformas y de las redes son un mundo nuevo por conocer.

A medida que las normas gubernamentales se van flexibilizando los restaurantes tradicionales regresan al mercado, mas establecimientos prestan el servicio a domicilio y se incorporan con estrategias de marketing más agresivas tratando de reconquistar sus mercados, por lo que los negocios que se iniciaron durante la pandemia se ven amenazados al tener una disminución de sus ventas, quienes consideran que no volverán a utilizar las plataformas, tal vez estén destinados a desaparecer.

Los negocios que se van adaptando a la utilización de las plataformas, escuchan a sus clientes y mantienen la calidad del producto y del servicio, no solo permanecerán en el mercado, sino tendrán la posibilidad de crecer en el mediano plazo. Cada vez, hay un mayor número de demandantes de bienes y servicios que realizan sus compras a través de la red, utilizando las diferentes plataformas. Al mismo tiempo las diferentes plataformas utilizan el marketing 
digital para atraer un mayor número de usuarios, lo que les garantizaría un mayor número de empresas que desearían utilizarlas.

Podríamos señalar que el futuro de los pequeños negocios de alimentos esta en las redes.

\section{References:}

1. Alejo, $\mathrm{H}$ (12 de junio de 2020) $15 \%$ de bares y restaurantes de Tabasco no volverían a abrir debido a crisis económica por Covid-19. Recuperado de http://xeva.com.mx/nota.cfm?id=108555\&t=15-debares-y-restaurantes-de-tabasco-no-volverian-a-abrir-debido-a-crisiseconomica-por-covid19.

2. Instituto Nacional de Estadística, Geografía e Informática (2013) Encuesta Anual de Servicios Privados No Financieros 2013. Obtenido de

http://internet.contenidos.inegi.org.mx/contenidos/Productos/prod_se rv/contenidos/espanol/bvinegi/productos/nueva_estruc/70282507154 7.pdf

3. López, R (20 de marzo de 2020). Tabasco: cierran restaurantes para evitar contagios de coronavirus. Recuperado de https://www.jornada.com.mx/ultimas/estados/2020/03/20/tabascocierran-restaurantes-para-evitar-contagios-de-coronavirus-9097.html

4. Méndez, M (2013) GIZMODO. Obtenido de https://es.gizmodo.com/los-12-tipos-de-personas-que-utilizan-redessociales-480499781

5. Meneses, C. (2017) La importancia de una plataforma digital alineada a tu negocio. Recuperado de https://comunidad.iebschool.com/cristianmeneses/2017/05/14/laimportancia-de-una-plataforma-digital-alineada-a-su-negocio/

6. Pérez, J. (6 de mayo de 2020). Restaurantes de Villahermosa solo entregarán a domicilio. Recuperado de https://www.elheraldodetabasco.com.mx/local/restaurantes-devillahermosa-solo-entregaran-a-domicilio-5197199.html

7. Pico-Versoza, L. M. (2017). El emprendimiento por necesidad, una ventana hacia el desarrollo de oportunidades de negocios. INNOVA Research Journal, 2(1), 131-136. DOI: 10.33890/innova.v2.n1.2017.133

8. Sixto, J. (2016) Fundamentos de Marketing Digital. Salamanca, España. Comunicación Social. Ediciones y publicaciones. 